\title{
EDDY CURRENT THERMOGRAPHY FOR RAIL INSPECTION
}

\author{
Torane Vaibhav*, Krishnan Balasubramaniam ${ }^{* *}$, Renil Thomas**, A. Chandra Bose* \\ ${ }^{*}$ Department of Physics, National Institute of Technology, Tiruchirapalli-620015 \\ ${ }^{* *}$ Centre for Non Destructive Evaluation, Indian Institute of Technology Madars-600036 \\ Email: vaibhav.torane@gmail.com,ktrenil@gmail.com,acbose@nitt.edu
}

\begin{abstract}
With the tendency of the railway transportation into heavy haul, more frequent usage of rail tracks and increased axle load, the problem of surface damage and fatigue is dominant on rails. Rolling contact fatigue (RCF), which leads to crack formation in rail track heads, is becoming a growing concern in the transportation industry. It occurs on or very close to the rail head surface, and is a significant cause of rail failure. The detection of cracks in RAILS is a critical requirement in the Railway industry. Cracks, if undetected will lead to rail fractures and consequently may lead to catastrophic accidents. Thus with the development and operation of high speed trains, condition based maintenance and monitoring becomes an important approach for the improvement of reliability and safety of rail transportation. Eddy Current Thermography (ECT) is an emerging NDT method especially for conductive material like rails which combines the advantages of eddy current testing and IR Thermography. Due to electrical conductivity, thermal conductivity and high permeability of rails, ECT is very suitable for its damage detections. This technology describes an inspection methodology which utilizes scanning induction Thermography in which coil is excited by a high frequency alternating current and induced eddy current are generated in sample placed near the coil. The thermographic camera is arranged to capture data indicative of a thermal response resulting from the flow of electrical current through the copper coil which scans the rail track. A computer system is configured to process the data from the thermographic camera to generate an indication of a presence of a discontinuity in the rails.
\end{abstract}

Keywords: Eddy Current, Thermo-Mechanical, Scanning Induction Thermography.

\section{Introduction}

One of the major problems that railroads have faced since the earliest days is the failures in the track during the service period. As is the case with all modes of high speed travel, failures of an essential component can have serious consequences. There are several factors that can influence the expected service life of rail, such as track maintenance programs, loading at the wheel-rail interface and rail stresses [1]. Rail tracks are subjected to intense stresses, deformation and wear. With the tendency of railway transportation into heavy haul and high speeds, the problem of surface damage due to wheel- rail rolling contact fatigue becomes more and more serious [2]. Rolling contact fatigue (RCF) is the process of fracture under repeated loading caused due to rolling or sliding contact. In rails, this phenomenon occurs due to cyclic loading of the material, occurring on the rail surface in the form of shelling and squats at the surface and sub- surface. The rolling contact between the rail surface and the wheel has high efficiency due to the small area of contact over which the load is transmitted. But due to this, the stress on the rolling contacts is relatively large [3]. Stress concentration at the contact area between the rail and wheel causes the initiation of RCF cracks. The stresses that are generated at the contact area are governed by the rail and wheel geometry at the contact patch, traction forces and existing conditions of the rail and wheel [4]. Under conditions of high friction, high contact stress and higher cycles of rotation (wheel over the rail), deformation of the wheel and rail metal in the direction of applied stress occurs [5].RCF cracks develop from the surface and usually propagate initially with a shallow angle and then, grow steeper inside. The cracks are formed along the shear planes on the top surface of the rail head. Different types of RCF cracks have been known to occur in rails such as: rail gauge corner cracking, shelling, transverse rail defects and squats [6]. 


\subsection{1/qirt.2016.138}

The safety and reliability of a railway system primarily depends on the stability of the critical components and their freedom from flaws. Crack initiation and propagation due to RCF is a matter of concern in the railway industry. If undetected, it will lead to rail fractures and consequently may lead to catastrophic accidents. Hence, a fast and effective method of inspecting rails, not only after the manufacturing stage, but also for online monitoring is mandatory.

In this paper, Non Destructive Testing (NDT) is implemented for the inspection of rail track. Features from the temperature distributions of the Eddy Current Thermography (ECT) investigations were extracted to gain quantitative information about the cracks in the rail sample. The reminder of the paper is organized as follows: Section 2 provides a background on the conventional NDT techniques that are implemented for rail inspection, laying emphasis on ECT technique and its advantages. Section 3 presents the physics and methodology behind ECT followed by section 4 , which entails the experimental procedure, results and analysis followed for the detection of artificial cracks introduced in the rail sample using ECT. The paper concludes with a discussion of work and its implication for online railway track inspection.

\section{Background}

Research on the application of nondestructive testing (NDT) methods for detection of defects in rails has been performed over the last 100 years. Although Ultrasonic testing has proved an excellent tool in the detection of cracks in the interior of the rail cross-section, they do suffer from a number of drawbacks, the major ones being;

- As always with conventional UT, acoustic coupling between the probe and the rail must be optimized for the technique to work well. So an acoustic couplant must be introduced, but even with couplant problems can be encountered where the surface of rail is damaged.

- The existing Ultrasonic systems are inadequate for detection of RCF defects because of the ultrasonic dead zone.

- $\quad$ There is lack of sensitivity to shallow, surface breaking defects. This means surface defects due to RCF cause shadowing or blocking of the ultrasonic wave preventing detection of deeper defects [6].

In addition, electromagnetic non-destructive testing methods are usually used in rail regular inspection. Alternating Current Field Measurement (ACFM) have a good potential to detect RCF cracks with maximum operating lift-off , but is not sensitive for sub surface defect and it is also difficult to identify multi-cracks[7].Electromagnetic acoustic transducers (EMATs) can generate the surface wave to detect the RCF in rail at a small standoff distance, which is a non-contact technique with high speed potential but lift-off is the limitation[8].Other noteworthy innovation include the development of low frequency eddy current (LFEC) techniques, designed to locate deep defects. This technique has the ability to penetrate the rail, along with the feature that LFEC waves are immune to longitudinal defects but are interfered with by transverse defects and the data is generated indicating the presence of transverse anomaly hidden under a horizontal crack [9].

Clearly the area of the rail defect detection has received much attention in the last decade, especially with regard to RCF initiated cracks. Currently combinations of techniques are needed for full assessment of the rail; one of which is a typical combination of Eddy Current Testing and InfraRed thermography (ECT).

In Pulse eddy current thermography (also known as induction thermography), a short burst of electromagnetic excitation is applied to the material under inspection, inducing eddy currents to flow in the material. Where these eddy current encounter a discontinuity they are forced to divert, leading to areas of high and low eddy current density. High current density areas experiences higher level of Joule heating, thus the defect can be identified from the IR image sequence both during heating period and during cooling. In addition, the continuing development of IR cameral technology has led to increase in spatial resolution, frame rate and temperature sensitivity [2]. These improvements have allowed thermography to develop from a qualitative overall inspection technique which is supplemented by other NDE technique like Pulse Eddy Current, to standalone technique able to supply quantitative information through the acquisition and analysis of image sequence [8].

The major advantages of Eddy Current Thermographic inspection over other technique are as follows;

- Non-contact, fast and high resolution.

- Thermography is applicable to wide range of materials and is able to inspect large area in short time, though selection of the optimal excitation for chosen application is important. 


\subsection{1/qirt.2016.138}

- Due to electrical conductivity, thermal conductivity and high permeability of rail ECT is very suitable for its damage detections.

\section{Methodology}

Scanning Induction thermography, a rapid, non-contact and non-destructive technique is used to test the specimen. Induction heating is accomplished by using an electromagnetic induction coil through which a current at specific frequency (typically $50-500 \mathrm{kHz}$ ) is applied. Whenever AC current passes through an electromagnetic coil with a certain frequency of excitation (here settled to $234 \mathrm{kHz}$ ), a varying magnetic field is developed around the coil. When this electromagnetic field is applied to conductive material placed near it, an electro-magnetic force will generate which induces a current to flow through it. This induced secondary current is called eddy current [4].In this study rail sample is kept stationary and a relative motion is given to the coil such that the relative movement is independent of the image recording with the infrared camera. As the coil scans the sample, eddy current are induced in the sample which causes a rise in the surface temperature of the sample and presence of defect will disturb the eddy current density distribution around it .The introduction of crack in the eddy current path results in diversion of eddy current around the tips of crack causing an increase in current density and resultant hot spot at the tip, whereas cold spot at the center of the crack where the eddy current density is low. The thermal response of the material is recorded by the IR Camera for the detection, characterization and sizing of anomalies in materials, thermal distribution pattern analysis is conducted by ALTAIR software [5].Fig.1 shows the hot spots where eddy current density is high due to diversion of eddy current path at the tips of the slot.

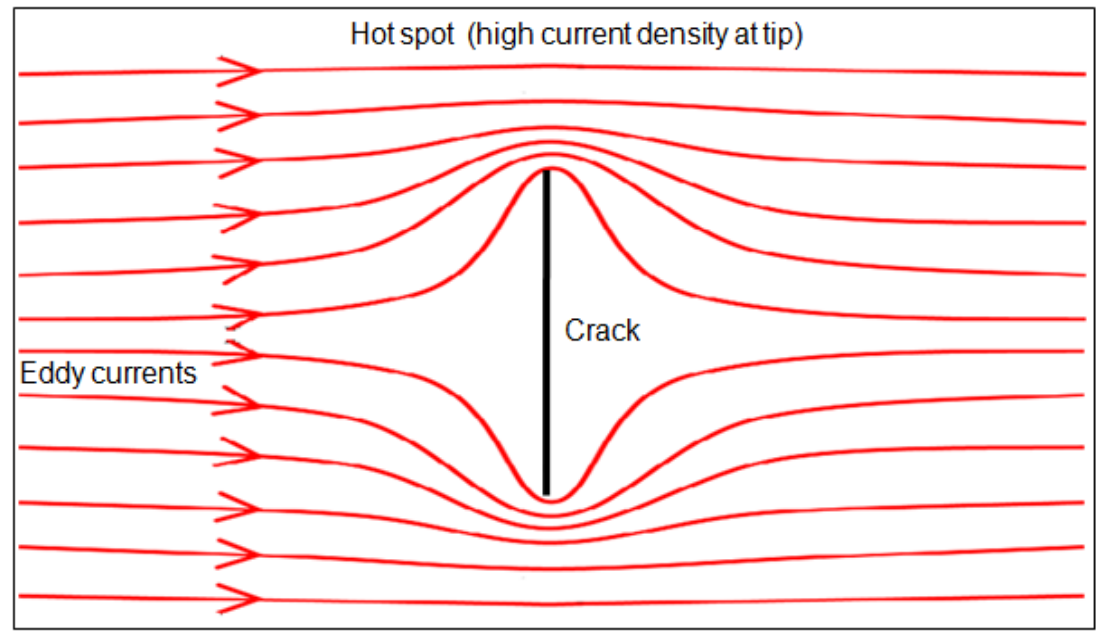

Fig.1. Schematic of theoretical eddy current distribution around the crack.

\section{Experimental Studies}

\subsection{Test set-up}

Experiments in this work are carried out with active thermography technique by keeping the induction coil and IR camera on the same side. A precision solid state induction heating equipment device Ameritherm INC is used for induction heating, with a maximum excitation power of $1 \mathrm{~kW}$ and excitation frequency range $150-400 \mathrm{kHz}$. The excitation frequency is settled at $234 \mathrm{kHz}$. The heating process is quick and clean, the rail sample to be heated is placed near to induction heating coil made of hollow copper tubing. The excitation coil has high conductivity and is $5 \mathrm{~mm}$ in diameter. Fig.2. (a) shows the schematic for the setup while (b) shows the experimental setup. The coil scans the rail for the detection of any defects. The defect are artificially introduced in the rail sample by electrical discharge machining (EDM), crack dimension being (length $\mathrm{x}$ width $\mathrm{x}$ depth) $25 \mathrm{~mm} \times 1 \mathrm{~mm} \times 4 \mathrm{~mm}$. 
(a)

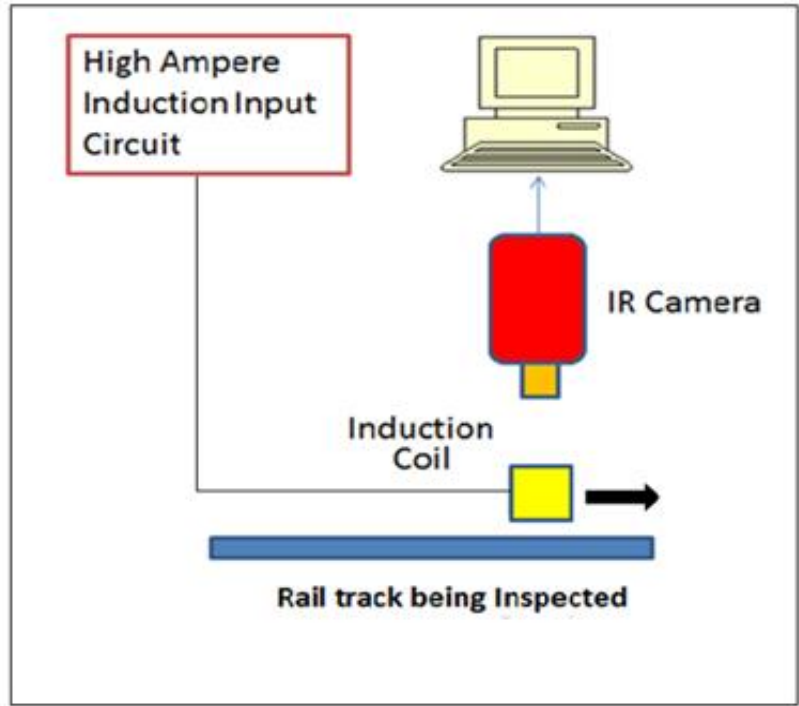

(b)

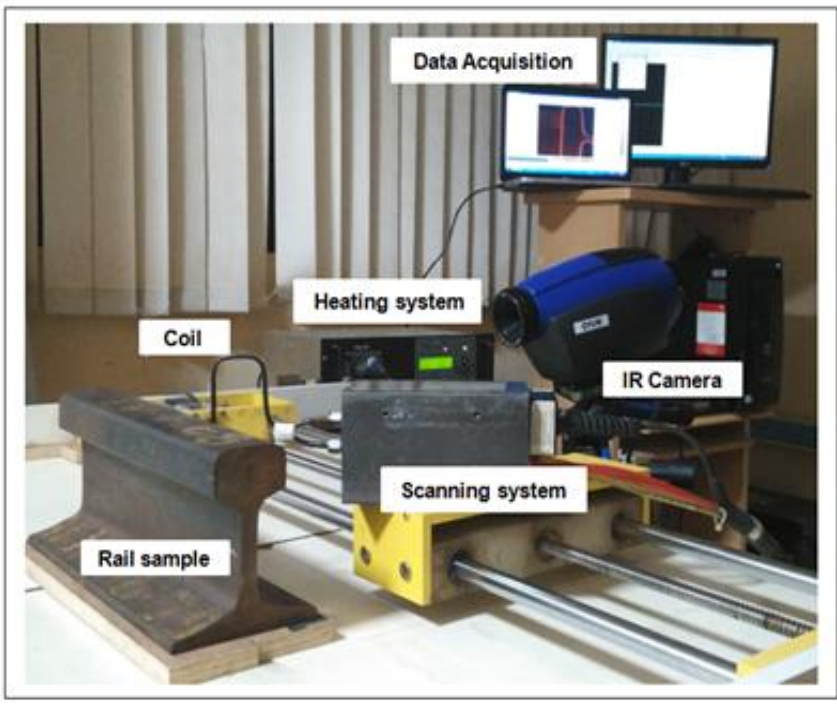

Fig.2. (a) Schematic setup diagram (b) Experimental setup

The infrared system FLIR SC5000 is used to record the temperature changes. It is equipped with a cooled Indium Antimonide ( $\mathrm{InSb}$ ) detector that allows the smallest of temperature differences to be seen, has a sensitivity of $20 \mathrm{mK}$ and maximum full frame rate of $383 \mathrm{~Hz}$. It operates in medium waveband of the 2.5 to $5.1 \mu \mathrm{m}$. Indium Antimonide (InSb) focal plane array delivers an outstanding frame rate while keeping excellent linearity and sensitivity. The scanning data is captured by active reflection thermography and the radiation emitted by specimen as the coil scans it are collected by IR camera which are sampled using commercial thermography software ALTAIR. This thermal analysis software can be coupled with any thermal camera to deliver superior thermal images with a wide dynamic range and high speed acquisition capabilities. Thermographic data is imaged at a rate of $50 \mathrm{fps}$ (frames per second).

The tests reported in this paper, are studied for eight different cases by varying the current from $250 \mathrm{~A}$ to $400 \mathrm{~A}$ with an increment of $50 \mathrm{~A}$ during each test and scanning at $40 \mathrm{~mm} / \mathrm{s}(1 \mathrm{X})$ and $80 \mathrm{~mm} / \mathrm{s}(2 \mathrm{X})$ respectively, the same is optimized for the defect detection. The coil is moved slowly enough over a rail sample to allow significant heating of the area under inspection using a step motor and motion controller device.

\subsection{Experimental result and analysis:}

Settling the excitation frequency at $234 \mathrm{kHz}$ eight different cases are studied by varying current and scanning speed, the same is optimized for the defect detection. Scanning speed of $40 \mathrm{~mm} / \mathrm{s}(1 \mathrm{X})$ and $80 \mathrm{~mm} / \mathrm{s}(2 \mathrm{X})$ is used to scan the sample by varying the amperage.Fig. 3 shows the schematic of five points marked along the edge of crack for surface temperature analysis as the coil just passes the point and scans it. The visualization and mapping of the temperature distribution due to the eddy current interaction with defect is analyzed using ALTAIR - thermal analysis software. Thermal image is captured by camera and analysis of selected points along the crack in ALTAIR software is shown in the Fig.4, while temperature vs. time graph is plotted for the five selected points in the Fig.5. The points 1 and 5 are the tip of the crack where the eddy current density is high thus hot spot occurs at 1 and 5 respectively as the coil scans, whereas the points $2,3,4$ are along the edge in the mid of crack where the density of eddy current is low and hence cold spots occurs at these points. 


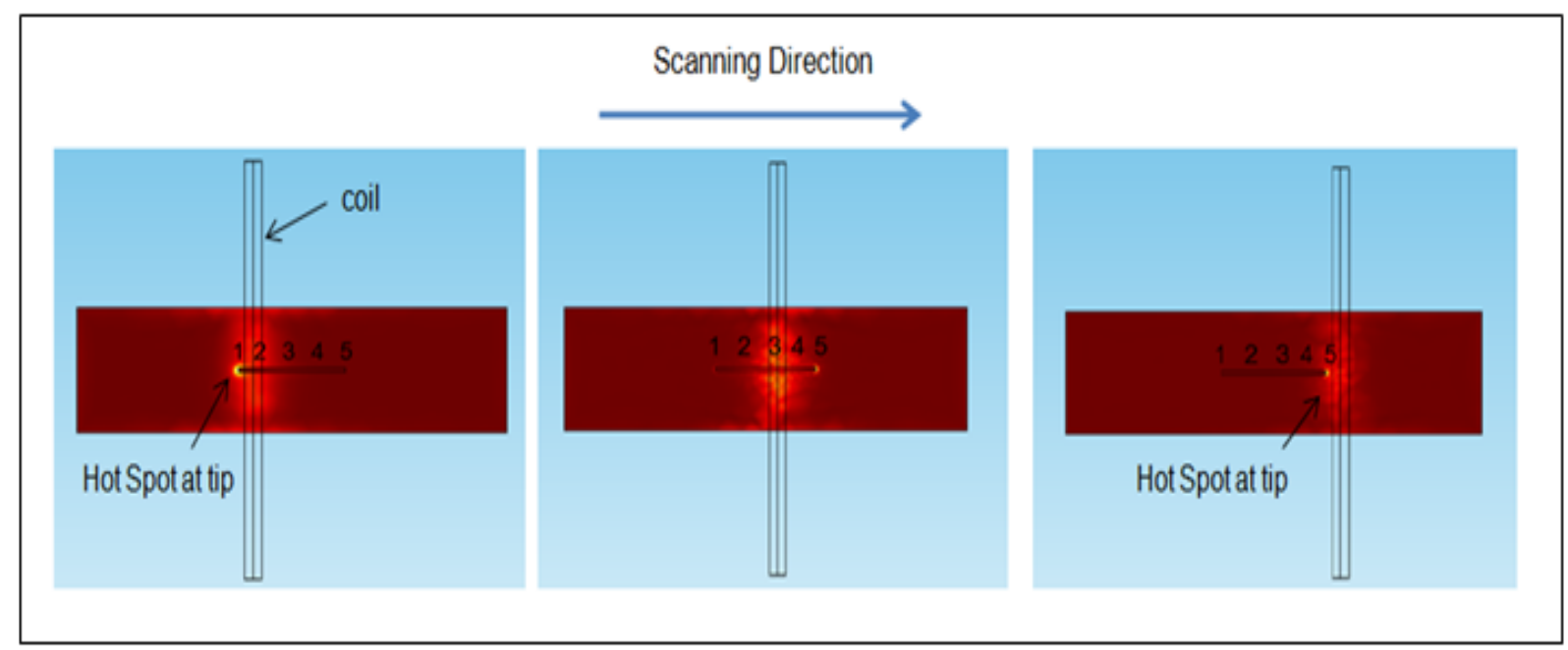

Fig.3.Schematic of coil scanning the rail sample for detection of hot spots and cold spots along the crack.

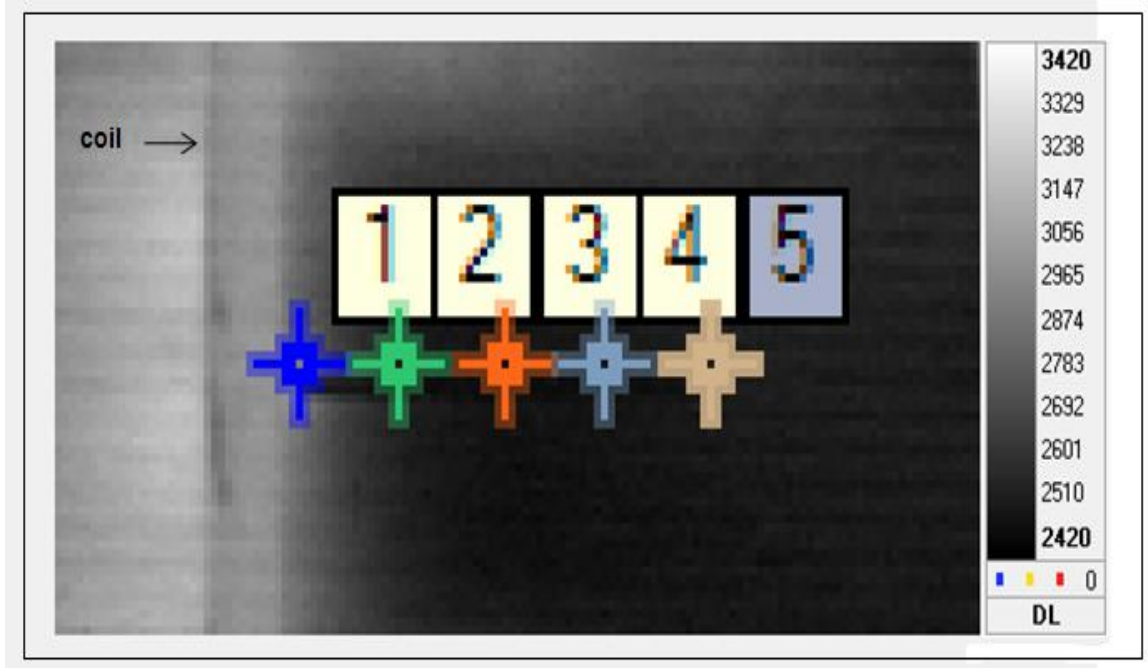

Fig.4. Thermal Image analysis using ALTAIR software

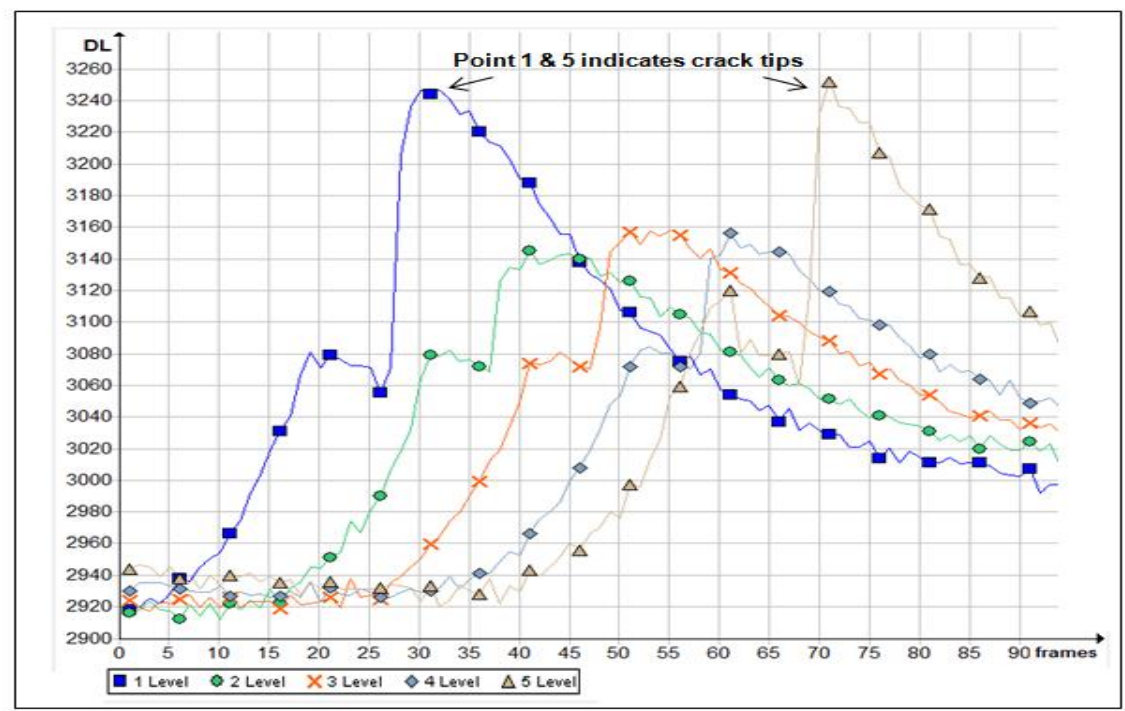

Fig.5. Temperature-Time graph obtained for five points along the crack, shows high temperature at tips. 


\subsection{1/qirt.2016.138}

The thermal responses were analyzed for these points along the crack using ALTAIR.Fig.6 (a)shows the temperature responses of five points for different amperage at speed 1X. A high current density distribution contributes a greater Joule heating which results in high heat generation at the crack tip, thus hot spots occur at points 1 and 5.Thermal contrast at the tip is visible more clearly due to the high current density at the tips. Thermal contrast on the surface of sample is the temperature difference taken between the two points on the surface of the sample at any time step, here average temperature of point 1 and $5\left(T_{\text {tip }}\right)$, which has the highest temperature and the average temperature of points 2,3 and 4 ( $\left.T_{\text {center }}\right)$ which has the lowest temperature. The computation of thermal contrast is given by,

$$
\Delta \mathrm{T}=\mathrm{T}_{\text {tip }}-\mathrm{T}_{\text {center }}
$$

Fig.6(b) Shows the plot of thermal contrast for the different amperage at speed 1X.SimilarlyFig.7 (a) shows the thermal responses of points along the crack when the coil just passes the points, while (b) shows the thermal contrast for the different amperage at speed $2 X$.

(a)

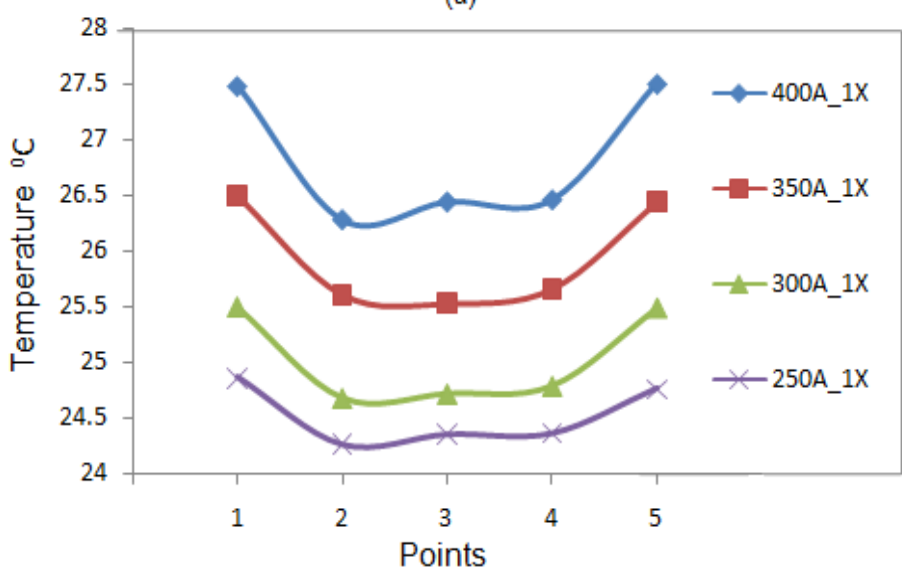

(b)

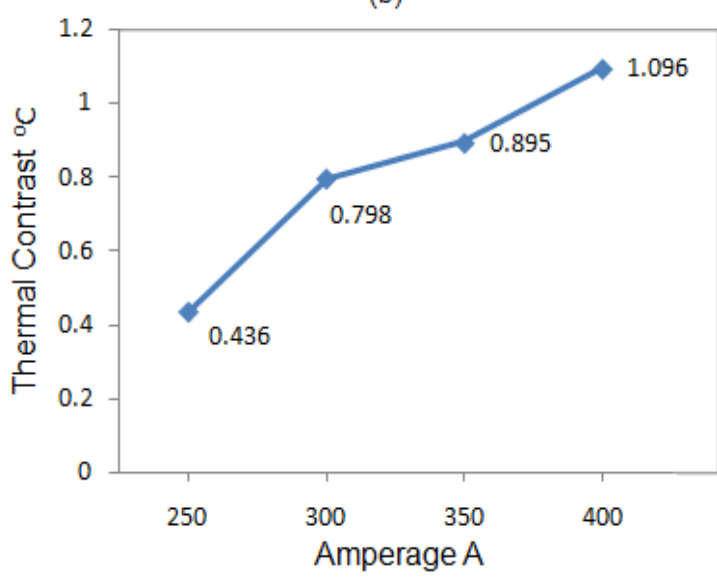

Fig.6. (a) Temperature responses of five points along the crack for different amperage at $1 X$ speed (b) Thermal contrast at different amperage for $1 X$ speed.

(a)

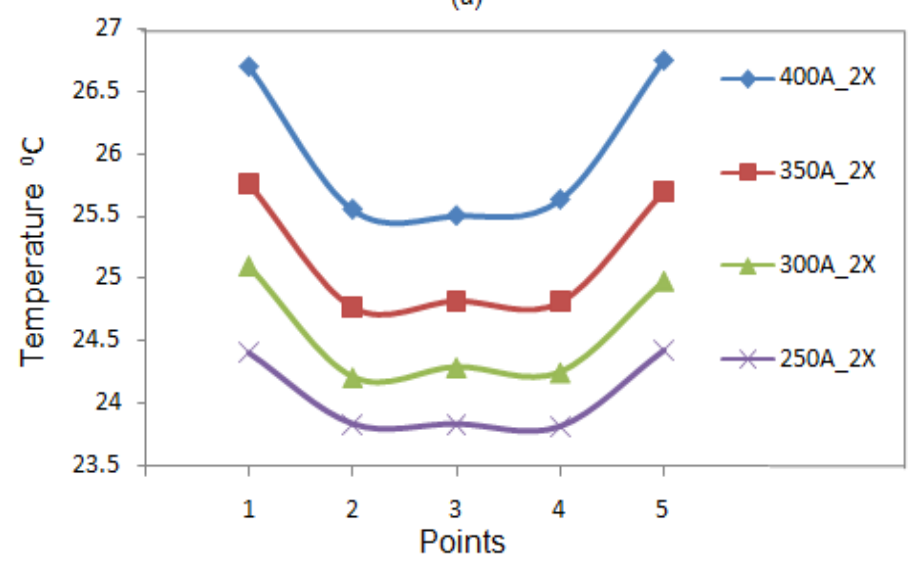

(b)

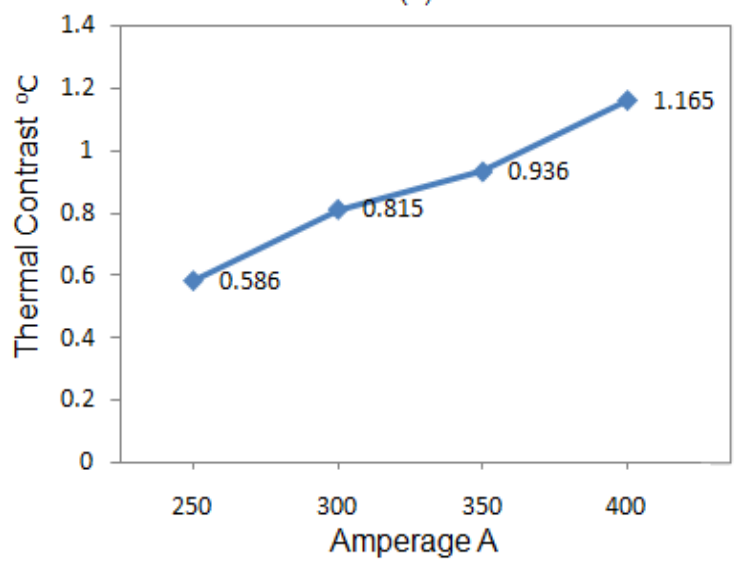

Fig.7. (a) Temperature responses of five points along the crack for different amperage at $2 X$ speed (b) Thermal contrast at different amperage for $2 X$ speed.

The Comparison plot for the thermal responses of five points at scanning speeds $1 \mathrm{X}$ and $2 \mathrm{X}$, for different amperage is shown in Fig.8. The surface temperature profiles can be correlated for the following cases;

(a) 250A_1X\&300A_2X,

(b) 300A_1X \& 350A_2X, 


\subsection{1/qirt.2016.138}

(c) 350A_1X \& 400A_2X

Thus by increasing the current and scanning at twice the speed would result into similar temperature profile and thermal contrast.

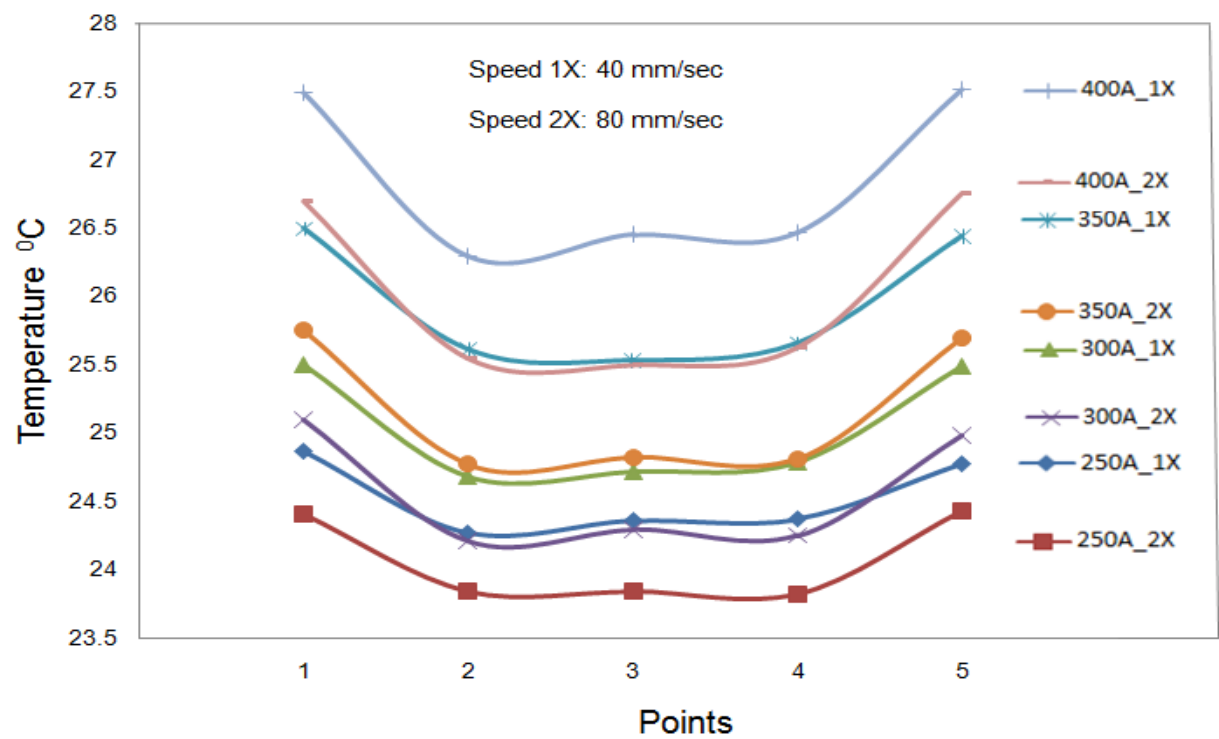

Fig.8. Comparison plot for thermal responses of five points for scanning speeds $1 X \& 2 X$, at different amperage.

\section{Discussion and Conclusion}

Although Scanning induction thermography or PEC thermography is capable of dynamic measurement, certain factors limit the deployment of speed. Also the scanning coil needs to move slowly enough to allow significant heating of area under inspection before imaging. Here two scanning speeds $40 \mathrm{~mm} / \mathrm{s}(1 X)$ and $80 \mathrm{~mm} / \mathrm{s}(2 X)$ were used to ensure sufficient heating and their effect at different currents is correlated to obtain similar temperature profiles.

In this work, the scanning induction thermography technique was used to detect the artificial cracks introduced in the rail sample. During experiment, it was proved that the presence of the defects in the material can influence the thermographic distribution when the sample in heated and current density is found to be concentrated around the crack tip which resulted in hot spot and cold spots areas. The crack detection carried at different scanning speeds (1X and $2 X)$ and at different amperage which showed the correlation between temperature profiles during experimenting at different cases. The thermal contrast was calculated and plotted by taking the difference between mean of maximum temperature and mean of minimum temperature of the points along the crack considered for thermal analysis. Thermal contrast at the tip is visible more clearly due to the high current density at the tips.

\section{REFERENCES}

[1] John Wilson, Guiyun Tian, Ilham Mukriz and Darryl Almond, PEC Thermography for imaging multiple cracks from rolling contact fatigue, NDT \& E International, Vol. 44, Pages 505-5, 2011.

[2] Abidin I.Z, Tian G.Y and Almond, Quantitative evaluation of angular defects by Pulsed eddy-current Thermography,NDT \& E International, Vol. 43, Pages 537-546, 2010.

[3] Ruizhen Yang, Bin Gao, Gui Yun Tian , Lateral heat Conduction based eddy current Thermograph for detection of parallel cracks and rail tread oblique cracks, Measurement, Vol.66, Pages 54-61, 2015.

[4] M.N. Libin, Krishnan Balasubramaniam, B.W. Maxfield and C.V.Krishnamurthy, Modeling and Simulation for crack detection in Aluminium components using tone burst eddy current thermography, Proceedings of National Seminar \& Exhibition on Non Destructive Evaluation, 2014. 


\subsection{1/qirt.2016.138}

[5] Louaayou, M. N. Nait-Said and F. Z. Loui, 2D Finite element method study of the stimulation induction heating in synchronic Thermography NDT, NDT \& E International,Vol. 41, Pages 577-58, 2008.

[6] Jianping Peng, Gui Yun Tian,Li Wang, Yu Zhang,et al., Investigation into eddy current pulsed thermography rolling contact fatigue detection and characterization, NDT \& E International,Vol.74, Pages 72-80, 2015.

[7] Biju N, N. Ganesan, C.V. Krishnamurthy and Krishnan Balasubramaniam, Frequency optimization for eddy current Thermography, NDT \& E International, Vol. 42, Pages 415-420, 2009.

[8] Tian G. Y, J. Wilson, L. Cheng, D.P. Almond, E. Kostson and B. Weekes, Pulsed eddy-current Thermography and applications, Lecture Notes in Electrical Engineering, Vol. 96, Pages 205-231, 2011.

[9] Cannon D.F, Edel K.O, Grassie S.L and Sawley K., Rail defects an overview, Fatigue \& Fracture of Engineering Materials \& Structures, Vol. 26, Pages 865-86, 2003.

[10] Vrana J, Goldammer M, Baumann J, Rothenfusser M and Arnold W, Mechanisms and models for crack detection with induction thermography, Review of Quantitative Nondestructive Evaluation, Vol. 27, Pages 47582, 2008.

[11] Nicholson G.L, Kostryzhev A.G, Hao X.J and Davis C.L , Modelling and experimental measurements of idealized and light-moderate RCF cracks in rails using an ACFM sensor, NDT \& E International, Vol. 44,Pages 427-37, 2011. 